\title{
Evaluación de la exposición a selenio en Los Altos de Jalisco, México
}

\author{
Roberto Hurtado-Jiménez, PhD, (1) Jorge Gardea-Torresdey, PhD. ${ }^{(2)}$
}

\author{
Hurtado-Jiménez R, Gardea-Torresdey J. \\ Evaluación de la exposición a selenio \\ en Los Altos de Jalisco, México. \\ Salud Publica Mex 2007;49:3 I2-3I5.
}

\section{Resumen}

Objetivo. Evaluar la exposición a selenio (Se) vía agua potable en los habitantes de Los Altos de Jalisco. Material y métodos. Se determinó la concentración de Se en 125 pozos y se estimaron los niveles de exposición a Se en bebés, niños y adultos. Resultados. La dosis de exposición y la ingestión de Se vía agua potable variaron en los siguientes rangos: a) bebés: I.3-6.7 $\mu \mathrm{g} / \mathrm{kg} / \mathrm{d}$ y $12.6-67.2 \mu \mathrm{g} / \mathrm{d}$; b) niños: 0.8-4.5 $\mu \mathrm{g} / \mathrm{kg} / \mathrm{d}$ y 16.8-89.6 $\mu \mathrm{g} / \mathrm{d} ; \mathrm{c}$ ) adultos: $0.6-3.0 \mu \mathrm{g} / \mathrm{kg} / \mathrm{d}$ y $33.6-179.2 \mu \mathrm{g} / \mathrm{d}$. Conclusiones. En este caso, la exposición a Se representa un riesgo potencial para la salud de la población, ya que en la mayoría de los casos es mayor que la recomendada por organismos internacionales de salud. Sin embargo, no es tan alta como para esperar la ocurrencia de selenosis.

Palabras clave: selenio; enfermedades de origen hídrico; agua potable; Los Altos de Jalisco; México
Hurtado-Jiménez R, Gardea-Torresdey J.

Evaluation of the exposure to selenium

in Los Altos de Jalisco, Mexico.

Salud Publica Mex 2007;49:3 I2-3I5.

\section{Abstract}

Objective. To evaluate the exposure to selenium in drinking water in Los Altos de Jalisco (Jalisco State Heights). Materials and Methods. The concentration of selenium was determined in 125 water wells, and the exposure doses to selenium were estimated for babies, children and adults. Results. The estimated values of the exposure doses to selenium and total intake of selenium were in the following ranges, respectively: (a) babies: 1.3-6.7 $\mu \mathrm{g} / \mathrm{kg} / \mathrm{d}$ and 12.6-67.2 $\mu \mathrm{g} / \mathrm{d}$; (b) children: 0.8-4.5 $\mu \mathrm{g} / \mathrm{kg} / \mathrm{d}$ and $16.8-89.6 \mu \mathrm{g} / \mathrm{d}$, (c) adults: $0.6-3.0 \mu \mathrm{g} / \mathrm{kg} / \mathrm{d}$ and 33.6-179.2 $\mu \mathrm{g} / \mathrm{d}$. Conclusions. The estimated exposure levels to selenium were higher than those recommended as optimum by international health organizations, representing a potential health risk. Nevertheless, estimated values are not high enough to produce selenosis.

Key words: selenium; waterborne diseases; drinking water; Los Altos de Jalisco; Mexico
$\mathbf{E}^{\mathrm{s}}$ 1 Selenio (Se) es un micronutriente esencial de gran importancia para los seres humanos, sobresaliendo su potente acción antioxidante y su capacidad para regular el mecanismo de la glándula tiroides. ${ }^{1,2}$

Las fuentes principales de Se son los alimentos y en algunos casos el agua. ${ }^{3}$ Los alimentos que contienen cantidades significativas de Se son: mariscos, carne roja (hígado y riñones), cereales (trigo), vegetales (ajo, cebo- llas, brócoli, hongos), huevos y lácteos. El alimento con mayor contenido de Se es la nuez de Brasil. ${ }^{4}$ La ingesta diaria recomendada (RDA, por sus siglas en inglés) es de 20, 30 y $55 \mu \mathrm{g} / \mathrm{d},(2.0,1.5$ y $0.8 \mu \mathrm{g} / \mathrm{kg} / \mathrm{d})$ para bebés, niños y adultos, respectivamente. ${ }^{5}$

Las deficiencias en la ingestión de Se pueden ocasionar diversos problemas, principalmente cardiacos y musculares. ${ }^{6}$ La exposición prolongada a dosis altas de

(I) Profesor-investigador. El Colegio de la Frontera Norte AC.

(2) Director del Departamento de Química. Universidad de Texas en El Paso, EUA.

Fecha de recibido: 16 de febrero de 2007 - Fecha de aceptado: 26 de junio de 2007 Solicitud de sobretiros: PhD Roberto Hurtado-jiménez. El Colegio de la Frontera Norte. Av. Insurgentes 3708 , Fracc. Los Nogales. 32350 Ciudad Juárez, Chihuahua, México Correo electrónico: rhurtado@colef.mx 
Se (mayor de $900 \mu \mathrm{g} / \mathrm{d}$ ), puede producir selenosis, cuyos síntomas principales son caída del cabello, fragilidad de las uñas, olor gárlico del aliento, elevada prevalencia de caries y problemas neurológicos. ${ }^{6}$

Existe evidencia de que la ingestión complementaria de 100-200 $\mu \mathrm{g} / \mathrm{d}$ de Se ayuda a prevenir diversas enfermedades, como algunos tipos de cáncer, ${ }^{7-9}$ trastornos cardiacos, ${ }^{2}$ infecciones virales ${ }^{10,11}$ y deficiencias de la tiroides. 12,13

Los altos niveles de selenio, flúor, arsénico y otros elementos potencialmente tóxicos en los acuíferos de Los Altos de Jalisco (LAJ) provienen de la infiltración de aguas geotérmicas profundas. ${ }^{14,15}$

El objetivo del presente estudio es evaluar, en forma preliminar, los riesgos potenciales a la salud de los habitantes de LAJ debido a la ingesta de agua potable con altos niveles de Se.

\section{Material y métodos}

Se muestrearon en dos ocasiones (noviembre de 2002 y octubre de 2003) todos los pozos que alimentaban las redes municipales de agua potable de 17 cabeceras municipales de LAJ. En cada uno de los 125 pozos se tomaron muestras por triplicado siguiendo los procedimientos convencionales (estabilización del flujo, filtrado, acidulado y refrigeración). ${ }^{16}$

La concentración de Se en el agua se determinó mediante espectrometría de emisión óptica con plasma acoplado inductivamente (ICP-OES, por sus siglas en inglés). El límite de detección es del orden de $1 \mu \mathrm{g} / 1{ }^{17}$

Las dosis de exposición a Se vía agua potable se estimaron utilizando la siguiente ecuación: ${ }^{18}$

$$
\begin{aligned}
& \mathrm{D}=\left(\mathrm{C}^{*} \mathrm{I} \mathrm{R}^{*} \mathrm{EF}\right) / \mathrm{BW} \\
& \text { donde, } \\
& \mathrm{D}=\text { dosis de exposición }(\mu \mathrm{g} / \mathrm{kg} / \mathrm{d}) \\
& \mathrm{C}=\quad \text { concentración de Se en el agua }(\mu \mathrm{g} / \mathrm{l}) \\
& \mathrm{IR}=\text { consumo diario de agua }(\mathrm{l} / \mathrm{d}) \\
& \mathrm{EF}=\text { factor de exposición (adimensional) } \\
& \mathrm{BW}=\text { peso corporal }(\mathrm{kg})
\end{aligned}
$$

Las dosis de exposición se estimaron para: a) bebés de 12 meses (10 kg); b) niños de seis años (20 kg); y c) adultos mayores de 25 años $(60 \mathrm{~kg})$. Los cálculos se basaron en un consumo de agua diario de $0.75,1$ y 2 litros para bebé, niño y adulto, respectivamente. ${ }^{14} \mathrm{El}$ factor de exposición se consideró como 1 (exposición cotidiana de largo plazo).

Los riesgos a la salud por la ingestión de Se, vía agua potable, se determinaron al comparar las dosis de exposición estimadas para los habitantes de LAJ con la $\mathrm{RDA}^{5}$ y los límites máximos tolerables (UL, por sus siglas en inglés) establecidos por el mismo instituto, ${ }^{5}$ que son de 60, 150 y $400 \mu \mathrm{g} / \mathrm{d}(6.0,7.5$ y $6.7 \mu \mathrm{g} / \mathrm{kg} / \mathrm{d})$ para bebés, niños y adultos, respectivamente.

\section{Resultados}

En el cuadro I se presentan los datos estadísticos correspondientes a los análisis químicos de Se practicados en el agua de los pozos muestreados. Las concentraciones promedio de Se en las cabeceras municipales de LAJ variaron entre 16.8 y $89.3 \mu \mathrm{g} / 1$. Las cabeceras municipales con las más altas concentraciones promedio de Se en los pozos muestreados fueron Teocaltiche (89.6 $\mu \mathrm{g} / 1)$, Jalostotitlán $(67.0 \mu \mathrm{g} / 1)$ y Unión de San Antonio $(63.9 \mu \mathrm{g} / 1)$. Los pozos con los niveles más altos de Se en LAJ fueron el número 6 de Teocaltiche $(121.9 \mu \mathrm{g} / \mathrm{l})$, el número 2 de San Miguel El Alto $(121.6 \mu \mathrm{g} / \mathrm{l})$ y el número 3 de Teocaltiche $(119.2 \mu \mathrm{g} / 1)$.

La dosis de exposición y la ingestión de Se vía agua potable, en las cabeceras municipales de LAJ, variaron en los siguientes rangos: a) bebés: $1.3-6.7 \mu \mathrm{g} / \mathrm{kg} / \mathrm{d}$ y 12.6-67.2 $\mu \mathrm{g} / \mathrm{d}$; b) niños: 0.8-4.5 $\mu \mathrm{g} / \mathrm{kg} / \mathrm{d}$ y $16.8-89.6$ $\mu \mathrm{g} / \mathrm{d}$; y c) adultos: $0.6-3.0 \mu \mathrm{g} / \mathrm{kg} / \mathrm{d}$ y 33.6-179.2 $\mu \mathrm{g} / \mathrm{d}$. Los valores promedio de ambas variables (para cada una de las cabeceras municipales) se muestran en la figura 1.

\section{Discusión}

La normatividad mexicana establece que la concentración máxima de Se en el agua embotellada es de 10 $\mu \mathrm{g} / 1 .{ }^{20}$ Aunque el Se no está regulado para el agua de la llave en México, se recomienda usar el estándar de la Organización Mundial de la Salud (OMS), ${ }^{21}$ que es 10 $\mu \mathrm{g} / \mathrm{l}$. Éste es un valor muy seguro ya que estaría muy lejos de causar toxicidad.

Los niveles de Se en el agua potable en $85 \%$ de las cabeceras municipales de LAJ (cuadro I) exceden el límite máximo de Se recomendado por la OMS, lo cual podría representar un importante problema de salud ambiental. Altas concentraciones de Se en el agua potable han sido también reportadas en la ciudad mexicana de Irapuato, donde se encontraron dos pozos con 218 y $223 \mu \mathrm{g} / \mathrm{l}^{22}$

La ingesta diaria de Se y las dosis de exposición estimadas para los habitantes de LAJ vía agua potable son muy altas (figura 1), ya que en la mayoría de las ciudades estudiadas rebasan los valores recomendados. ${ }^{5}$ Esto representa un riesgo potencial para algunas enfermedades secundarias, como náuseas, vómito y diarrea, principalmente para bebés y niños. Es importante que por lo menos niños y bebés no consuman agua de la llave, y que la supervisión de las autoridades sea tal que las empresas que venden agua de la llave purificada cumplan con la 

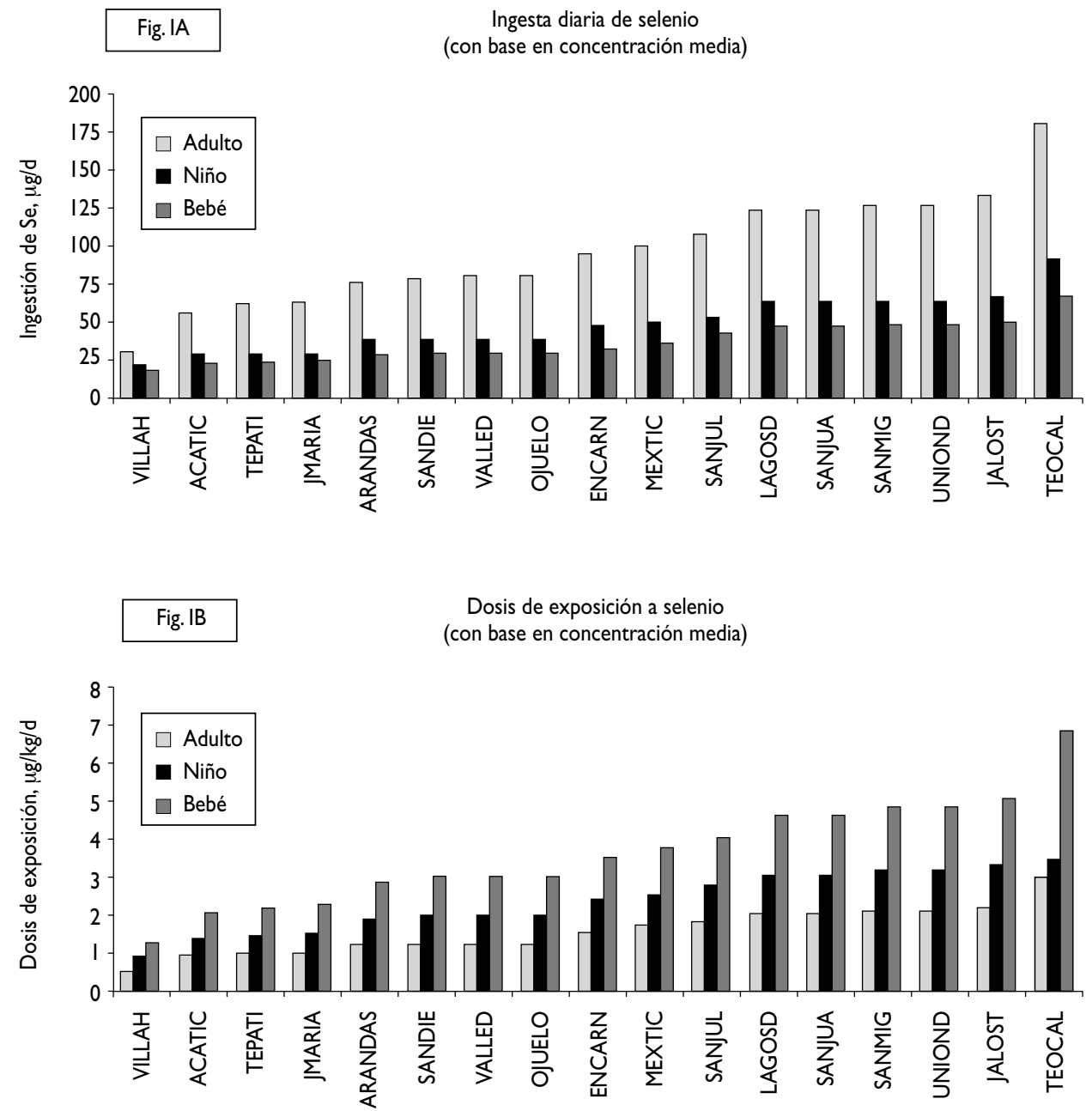

Figura I. Ingestión de selenio y dosis de exposición a selenio vía agua potable en Los Altos de Jalisco, México. 2002-2003

norma oficial vigente. En este estudio no se incluye la contribución de los alimentos a la ingesta total de Se. Esto significa que los niveles de exposición a Se son mayores y por lo tanto también los riesgos a la salud.

El riesgo de que se presenten casos de selenosis es prácticamente inexistente, ya que en ningún caso se alcanzan los límites máximos tolerables, aunque se tomara en cuenta la ingestión de Se en alimentos. En LAJ es muy difícil establecer los niveles tóxicos de Se, ya que existen otros contaminantes en el agua -como arsénico- con los que interacciona químicamente. Además, el Se podría estar contribuyendo a mitigar los efectos tóxicos del arsénico ${ }^{23}$ y otros contaminantes. Estudios epidemiológicos basados en la medición de niveles de elementos traza en sangre y orina podrían ayudar a confirmar dicha hipótesis. Se recomienda realizar estudios para determinar la contribución de los alimentos a la ingesta diaria de selenio.

\section{Agradecimientos}

Agradecemos el apoyo de las siguientes instituciones para la realización de este trabajo: Agencia de Protección Ambiental de los Estados Unidos (US EPA), Autotransportes Mezcala (ATM), El Colegio de la Frontera Norte y la Universidad de Texas en El Paso. 


\section{Cuadro I}

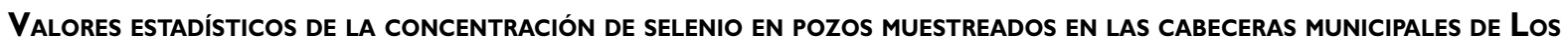
Altos de Jalisco, México, 2002-2003

\begin{tabular}{|c|c|c|c|c|c|c|}
\hline \multirow[t]{2}{*}{ Cabecera municipal } & \multirow[t]{2}{*}{ Población* } & \multirow[t]{2}{*}{ Pozos muestreados } & \multicolumn{4}{|c|}{ Concentración de Selenio $(\mu \mathrm{g} / \mathrm{l})$} \\
\hline & & & Promedio & Desviación estándar & Mínima & Máxima \\
\hline Acatic & 11005 & 4 & 27.9 & 8.6 & 20.5 & 40.2 \\
\hline Arandas & 39478 & II & 37.7 & 8.1 & 27.7 & 52.2 \\
\hline Encarnación de Díaz & 20772 & 6 & 46.2 & 27.9 & 21.0 & 94.4 \\
\hline Jalostotitlán & 21291 & 5 & 67.0 & 34.2 & 11.9 & 103.3 \\
\hline Jesús María & 7852 & 4 & 31.0 & 2.2 & 28.6 & 33.8 \\
\hline Lagos de Moreno & 79592 & 18 & 61.3 & 18.4 & 28.6 & 102.5 \\
\hline Mexticacán & 3603 & 4 & 49.7 & 37.6 & 2.0 & 88.2 \\
\hline Ojuelos de Jalisco & 9338 & 3 & 40.3 & II.I & 32.8 & 53.0 \\
\hline San Diego de Alejandría & 4749 & 2 & 39.5 & 0.7 & 39.1 & 40.0 \\
\hline San Juan de los Lagos & $424 I I$ & 10 & 61.6 & 30.2 & 11.8 & 98.0 \\
\hline San Julián & 12117 & 3 & 53.6 & 10.3 & 44.1 & 64.5 \\
\hline San Miguel el Alto & 21098 & 6 & 63.6 & 36.1 & 8.2 & 121.6 \\
\hline Teocaltiche & 21518 & 5 & 89.6 & 39.6 & 26.1 & 121.9 \\
\hline Tepatitlán de Morelos & 74262 & 27 & 29.4 & 16.1 & 11.4 & 75.7 \\
\hline Unión de San Antonio & 6317 & 2 & 63.9 & 39.4 & 36.1 & 91.7 \\
\hline Valle de Guadalupe & 4178 & 4 & 40.1 & 9.9 & 25.7 & 48.4 \\
\hline Villa Hidalgo & II 552 & II & 16.8 & 7.2 & 6.7 & 33.0 \\
\hline Total & 391133 & 125 & 45.1 & 27.1 & 2.0 & 121.9 \\
\hline
\end{tabular}

* Población correspondiente al Censo General de Población y Vivienda $2000^{19}$

\section{Referencias}

I. Rayman MP.The importance of selenium to human health. Lancet 2000;356:233-24I.

2. Brown KM,Arthur JR. Selenium, selenoproteins and human health: a review. Public Health Nutr 200 I;4(2B):593-599.

3. World Health Organization. Selenium in Drinking Water - Background document for development of WHO Guideline for Drinking Water. ReportWHO/SDE/WSH/03.04/I 3. Ginebra:WHO;2003.

4. National Institutes of Health. Dietary supplement fact sheet: Selenium. [Consultado: 22 febrero 2005]. Disponible en: http:/lods.od.nih.gov/ factsheets/Selenium_pf.asp.

5. Institute of Medicine. Selenium. En: Dietary reference intakes:Vitamin C, vitamin E, selenium, and carotenoids. Washington, DC: National Academy Press; 2000:284-324.

6.Agency for toxic substances and disease registry (ATSDR). Toxicological profile for selenium. US Public Health Service, US Department of Health and Human Services, Atlanta, Georgia. 2003.

7.Whanger PD. Selenium and its relationship to cancer: an update. British J Nutr 2004;91:I I-28.

8. Sanz Alaejos M, Díaz Romero FJ, Díaz Romero C. Selenium and cancer: Some nutritional aspects. Nutrition 2000;16:376-383.

9. Suárez de Ronderos MP. El papel del selenio y la vitamina E en la prevención y tratamiento del cáncer de próstata. Rev costarric salud publica 2004; I3(4): I- 14.

10. Rayman MPThe argument for increasing selenium intake. Proc Nutr Soc 2002;61:203-2I5.

I I. Ferencik M, Ebringer L. Modulatory effects of selenium and zinc on the immune system. Folia Microbiol 2003;48(3):417-426.

I2.Vrca VB, Skreb F, Cepelak I, Romic Z, Mayer L. Supplementation with antioxidants in the treatment of Graves' disease; the effect on glutathione peroxidase activity and concentration of selenium. Clin Chim Acta 2004;34I:55-63.
13. Köhrle J.The trace element selenium and the thyroid gland. Biochimie 1999;81:527-533.

14. Hurtado-jiménez R, Gardea-Torresdey J. Estimación de la exposición a fluoruros en Los Altos de Jalisco, México. Salud Publica Mex 2005;47:58-63. 15. Hurtado-Jiménez R, Gardea-Torresdey J.Arsenic in drinking water in the Los Altos de Jalisco region of Mexico. Rev Panam Salud Publica 2006;20(4):236-247.

16. United States Geological Survey. National field manual for the collection of water-quality data: US Geological Survey Techniques of water-resources investigations. [Consultado 12 octubre 2006]. Disponible en: http://pubs.water.usgs.gov/twri9A.

17. Perkin Elmer. Guide to inorganic analysis. [Consultado 12 octubre 2006]. Disponible en: http://las.perkinelmer.com/Content/ Manuals/ GDE_InorganicAnalysis.pdf.

18. Agency for toxic substances and disease registry. ATSDR Public Health Assessment Guidance Manual. US Public Health Service, U.S. Department of Health and Human Services, Atlanta, Georgia. 2005.

19. Instituto Nacional de Estadística, Geografía e Informática. XII Censo General de Población y Vivienda 2000. México: INEGI; 200 I.

20. Secretaría de Salud. Norma Oficial Mexicana: NOM 20I-SSAI-2002. Productos y servicios. Agua y hielo para consumo humano, envasados y a granel. Especificaciones sanitarias. México, DF: Diario Oficial de la Federación DLXXXIX 17, 18 octubre 2002:21.

2I.World Health Organization. Guidelines for drinking-water quality, Vol. I. Recommendations. 3a ed. Ginebra:WHO; 2004.

22. Wróbel-K K, López-de-Alba PL,Wróbe-Z K, López-Martínez L, Pantoja Villagómez LE. Selenium in municipal wells in the Irapuato Cityspectrofluorimetric determination with 2,3 diaminoaphthalene.Aquat Ecosyst Health Management 2001;4:209-2I3.

23. Chen Y, Hall M, Graziano JH, Slavkovich V, Van Geen A, Parvez F,Ahsan H.A prospective study of blood selenium levels and the risk of arsenicrelated premalignant skin lesions. Cancer Epidemiol Biomarkers Prev 2007; I6(2):207-2।3. 